\title{
Epstein-Barr virus-associated gastric ulcer mimicking gastric neoplasia: a case report
}

\author{
Adrian Catinean ${ }^{1}$, Radu Motocu ${ }^{2}$, Bogdan Fetica ${ }^{3}$, Anita Mezei $^{4}$
}

${ }^{1}$ Department of Gastroenterology, $1^{\text {st }}$ Medical Clinic, "Iuliu Hatieganu” University of Medicine and Pharmacy, ${ }^{2}$ Department of Surgery, County Emergency Hospital, ${ }^{3}$ Department of Pathology, "Ion Chiricuță" Institute of Oncology, ${ }^{4}$ Department of Gastroenterology, "Octavian Fodor" Regional Institute of Gastroenterology and Hepatology, ClujNapoca, Romania

\begin{abstract}
We describe a rare case of a severe gastric ulcer of a 66-year-old immunocompetent man who was associated with primary Epstein-Barr virus (EBV) infection and temporarily misdiagnosed with gastric lymphoma. A contrast-enhanced computed tomography (CT-scan) of the thorax, abdomen and pelvis followed by EUS revealed a neoplastic infiltrative-ulcerative semicircumferential gastric mass and a $1 \mathrm{~cm}$ large satellite adenopathy, being classified as T3N1M0 gastric neoplasia. The histological analysis of the $3 / 4$ distal stomach and lymph node sthat were resected contradicted the initial biopsy report, suggesting EBV gastric ulcer, etiology confirmed by the in situ hybridization technique.
\end{abstract}

Keywords: endoscopic ultrasound; gastric neoplasia; Epstein-Barr virus

\section{Introduction}

Epstein-Barr virus, known as the human herpes virus 4 , infects over $90 \%$ of the world's population by adulthood and establishes lifelong, latent infections [1]. EBV infection has been proven responsible for a wide range of acute or chronic disorders, as well as benign or malignant conditions. A study published by Lugmani et al stated that a certain number of gastric carcinomas are associated with EBV infection, mainly lymphoepithelial gastric cancer in the gastric corpus, without any distinct clinical features [2].

Herein, we report a rare case of EBV-associated gastric ulcer with diffuse atypical lymphoid hyperplasia of the gastric mucosa in an immunocompetent adult patient.

Received 07.05.2018 Accepted 21.08.2018

Med Ultrason

2019, Vol. 21, No 1, 96-98

Corresponding author: Anita Mezei, MD

Department of Gastroenterology, "Octavian

Fodor” Regional Institute of Gastroenterology and Hepatology, Cluj-Napoca, Romania

18/28 Grigore Alexandrescu Street 400529

Cluj-Napoca, Romania

E-mail: mezei anita@yahoo.com
The involvement of EBV infection in benign upper gastrointestinal disorders has not been well documented.

\section{Case report}

A 66-year-old Caucasian male patient sought medical care for insidious onset of fatigue, nausea, heartburn and epigastric pain for 1 month. His past medical history reported essential arterial hypertension, abdominal aortic aneurysm and benign prostatic hyperplasia. His previous medication included Telmisartan $40 \mathrm{mg} /$ day for blood pressure control. The physical examination merely revealed epigastric tenderness on palpation. Laboratory studies showed an elevated erythrocyte sedimentation rate of $30 \mathrm{~mm} / \mathrm{h}$ and $\mathrm{H}$. pylori stool antigen was negative. First esophagogastroduodenoscopy (EGD) unveiled $1.5 \mathrm{~cm}$ lesser curvature gastric ulcer, with a clean fibrinous base, irregular sharp edges and surrounding inflammation. Multiple biopsies were taken to rule out malignancy, revealing histologic evidence of chronic gastritis with mild activity and minimal atrophy, without $\mathrm{H}$. pylori infection. The patient was discharged with esomeprazole $40 \mathrm{mg} /$ day treatment. Due to the atypical 


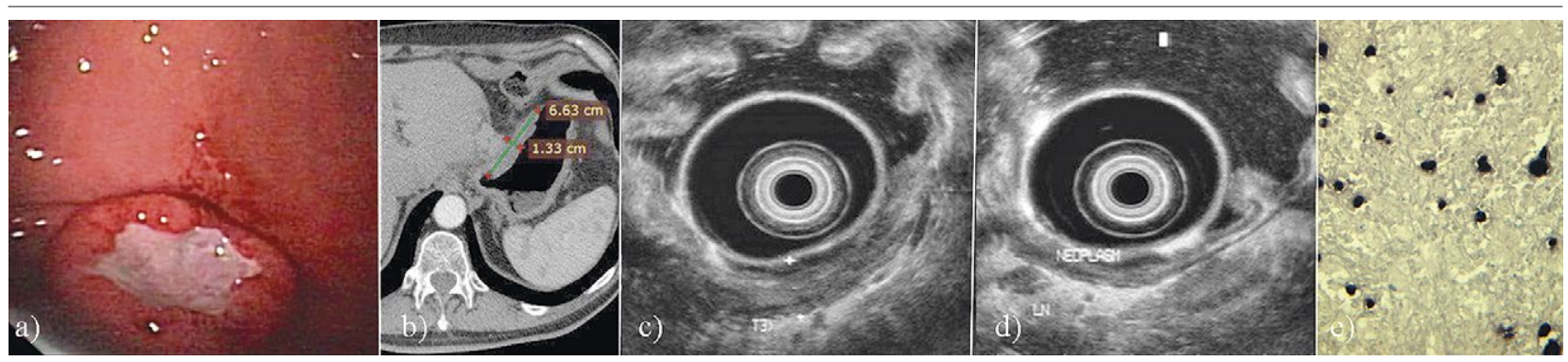

Fig 1. a). First esophagogastroduodenoscopy unveiled $1.5 \mathrm{~cm}$ lesser curvature gastric ulcer with a clean fibrinous base, irregular sharp edges and surrounding inflammation; b) Axial CT scan depicts inhomogeneous and thickened wall of the lesser curvature; c) Endoscopic ultrasound aspect of the gastric corporeal lesion with high suspicion for medullary pattern neoplasia with serosal invasion (irregularity of the fifth layer) T3 and d) positive lymph node on the lesser curvatureN1; e) Numerous large cells are positive for EBV in situ hybridization.

aspect of the ulcer (lymphoma-like) a second EGD after 6 weeks was performed. No clear macroscopic improvement was found (fig 1a) and biopsy mapping was performed once again. Immunohistochemical studies excluded malignancy: only $30 \%$ of the inflammatory cells expressed Ki-67, precluding lymphoma, cytokeratin ruled out epithelial malignancy and inflammatory cells showed positive staining for CD45 excluding lymphoepithelial lesions.

CT-scan of the abdomen revealed iodophile, inhomogeneous and thickened $(12 \mathrm{~mm})$ wall of the lesser curvature (fig $1 \mathrm{~b}$ ) and regional lymphadenopathy up to 1.5 $\mathrm{cm}$. The dose of esomeprazole was increased to $80 \mathrm{mg} /$ day. The third EGD, after three weeks revealed again no improvement and an EUS of gastric ulcerative mass was performed. A neoplastic-like infiltrative-ulcerative, semicircumferential gastric corporeal mass located between $48-54 \mathrm{~cm}$ from the dental arcade that infiltrated all layers of the gastric wall, invading the serousa was found. The thickness of the gastric wall was $11 \mathrm{~mm}$, with no perigastric ascites, which excluded peritoneal carcinomatosis. Image of a $1 \mathrm{~cm}$ large satellite adenopathy was related, presenting ultrasonography patterns advocating malignancy (hypoecogenic, round, sharp margins) (fig 1c,d).

Considering the lack of response to treatment and the abdominal CT and EUS results, despite the histopathological results, a multidisciplinary team speculated that the gastric lesion was highly suspicious for T3N1M0 gastric corporeal neoplasia. The patient underwent a distal partial gastrectomy (3/4), followed by Roux-en-Y gastric enteral anastomosis, accompanied by extended lymphadenectomy. He recovered from the surgical intervention uneventfully.

Pathology specimen revealed numerous large lymphocytes, some with typical morphology of the ReedSternberg cell, with marked proliferation index for Ki67, with the following immunohistochemical profile:
$\mathrm{CD} 30+, \mathrm{CD} 20+, \mathrm{LCA}+, \mathrm{CD} 3$-, CK AE1 / AE3 -. The morphological picture was strongly suggestive of EBV positive gastric ulcer. In situ hybridization technique was positive for EBV encoding region (EBER) (fig 1e). The perigastric lymph nodes were isolated without malignant aspect.

According to the clinical and pathological characteristics, a diagnosis of EBVs-associated gastric ulcer was rendered. No signs of recurrence were detected during the first 12 months of follow-up.

\section{Discussions}

EBV infection can lead to an extensive range of diseases varying in relation to the host's immune status. Mononucleosis is a self-limited disease caused by primary contact with EBV. Severe complications are rare and unpleasant events, usually involving different organ systems, rarely engaging the gastrointestinal tract. EBV was initially described only in lymphocytes infiltrating gastric tumours, its presence later being demonstrated in non-atrophic and non-metaplastic mucosa as well [3]. To present, the involvement of EBV infection in benign upper gastrointestinal disorders is not well documented. Case reports outline severe gastritis caused by acute EBV infection $[4,5]$ which can even mimic lymphoma [6]. According to Shukla et althe EBV infection was found in $70 \%$ of Indian peptic ulcer, sometimes associated with HP infection, which is negative in present case [7]. There are some experimental data regarding the enhanced inflammatory response in lab animals through mediators such as interleukin IL-8 and IL-1 $\beta$. This mechanism has not yet been proven in humans [8], even though EBV colonisation of digestive tract burdens the microbiota and immune system.

However, the corroboration of the clinical course, the serological results, the histological findings and the 
in situ hybridization study, yielded ample evidence that the illness was due to a primary response to EBV infection. It is important to identify EBV presence in gastric ulcer lesions irresponsive to PPI treatment, since it may resemble lymphoma.

At present, EUS is the most accurate imaging modality for the evaluation and staging of infiltrative lesions. Radial scanning revealed marked thickening of the gastric wall, with disruption of the layers and penetration of the serosa, an aspect suspicious for malignancy, such as in the present case. The clinical and morphological features of gastric lymphomas can resemble those of an ulcer. Because lymphomas tend to be submucosal and to ulcerate as the disease progresses, abnormalities of the stomach can be detected in up to $75 \%$ of patients. On the other hand, classic gastric ulcer does not usually infiltrate muscularis propria. Using elastographic assessment could have evidenced elements for differential diagnosis between the benign and malign aspect. The sensitivity of EUS FNA to the positive diagnosis of this type of lesion is small but targeting the lymph nodes could have been more useful in this case [9].

To conclude, it is important to test atypical ulcer lesions with neoplastic imaging features not responding to PPI therapy for EBV infection to prevent misdiagnosis and unnecessary invasive procedures.

\section{References}

1. Young LS, Rickinson AB. Epstein-Barr virus: 40 years on. Nat Rev Cancer 2004;4:757-768.

2. Lugmani YA, Linjawi SO, Shousha S. Detection of Epstein-Barr virus in gastrectomy specimens. Oncol Rep 2001;8:995-999.

3. Martinez-Lo'pez JL, Torres J, Camorlinga-Ponce M, et al. Evidence of Epstein-Barr virus association with gastric cancer and non-atrophic gastritis. Viruses 2014;6:301-318.

4. Zhang Y, Molot R. Severe gastritis secondary to EpsteinBarr viral infection. Unusual presentation of infectious mononucleosis and associated diffuse lymphoid hyperplasia in gastric mucosa. Arch Pathol Lab Med 2003;127:478-480.

5. Hisamatsu A, Nagai T, Okawara H, et al. Gastritis associated with Epstein-Barr virus infection. Intern Med 2010;49:2101-2105.

6. Tae CH, Lee JH, Min BH, Kim KM, Rhee PL, Kim JJ. Negative Biopsy after Referral for Biopsy-Proven Gastric Cancer. Gut Liver 2016;10:63-68.

7. Shukla SK, Prasad KN, Tripathi A, et al. Epstein-Barr virus DNA load and its association with Helicobacter pylori infection in gastroduodenal diseases. Braz J Infect Dis 2011;15:583-590

8. Cardenas-Mondragon MG, Torres J, Flores-Luna L, et al. Epstein-Barr virus association with peptic ulcer disease. Anal Cell Pathol (Amst) 2015;2015:164840.

9. Hawes RH, Fockens P, Varadarajulu S. Endosonography 3rd edition, Saunders; 2014;365-366. 